\title{
Abordagem do modelo transteórico no comportamento alimentar
}

\author{
Transtheoretical model approach in eating behavior
}

Natacha Toral ${ }^{1}$

Betzabeth Slater ${ }^{1}$
1 Departamento de Nutrição, Faculdadede SaúdePública,

Universidade de São Paulo. Av. Dr. Arnaldo 715 , Cerqueira César. 01246904. São Paulo SP. natytb@usp.br
Abstract The study of eating behavior has been much interest, as this is an essential element for the success of dietary interventions. In view of the complexity of the subject and the countless influences to which it is subject, it is suggested that an in-depth exploration of eating behavior determinants enhances the impact of programs promoting healthy dietary practices. Increasingly more frequent, the adoption of inadequate diets in Brazil and elsewhere in the world leads to questions about the impact of dietary interventions traditionally applied to population groups. M any nutritional education strategies are currently described in the literature; however, motivating people to change their eating habits is still a major public health challenge. Applying the transtheoretical model seems to have a promising role for an enhanced understanding of changes in eating habits that are targeted by dietary interventions. Strategies that are tailored to each step in these changes, as identified by this theory, can motivate people more effectively to adopt healthier eating habits.

Key words Eating habits, Theories, Transtheoretical model, Dietary interventions
Resumo 0 estudo do comportamento alimentar tem despertado grande interesse por se tratar de um elemento importante para o sucesso de intervenções nutricionais. Considerando-se a complexidade do tema e as inúmeras influências a que está submetido, sugere-se que o aprofundamento de pesquisas sobre os determinantes do comportamento alimentar possi bilite maior impacto nas ações de promoção de práticas alimentaressaudáveis. A adoção cada vez maisfreqüente de uma alimentação inadequada no Brasil e no mundo leva a um questionamento sobre $0 \mathrm{im}$ pacto das intervenções nutricionais tradicionalmente utilizadas em âmbito populacional. Diversas estratégias de educação nutricional são atualmente descritas na literatura; contudo, alcançar a motivação da população para uma mudança efetiva do padrão alimentar ainda é um dos grandes desafios para a saúde pública. $A$ aplicação do modelo transteórico parece ter um papel promissor em relação à melhor compreensão da mudança de comportamento alimentar, almejada nas intervenções nutricionais. Estratégias que en vol vam o direcionamento para cada estágio de mudança de comportamento, identificado segundo essa teoria, podem ser mais eficazes quanto à motivação dos indivíduos a adotar práticas alimentares mais saudáveis.

Palavras-chave Comportamento alimentar, Teorias, M odelo transteórico, Intervenções nutricionais 
Introdução

U ma busca crescente por maior compreensão do comportamento alimentar individual ou degrupos populacionais tem sido constantemente observada na literatura. Este comportamento corresponde às atitudes relacionadas às práticas alimentares em associação a atributos socioculturais, como os aspectos subjetivos intrínsecos do indivíduo ou próprios de uma coletividade, que estejam envolvidos com o ato de se alimentar ou com o alimento em si ${ }^{1}$. Destaca-se que, para o melhor entendimento do tema, é indispensável aprofundar o conhecimento dos determinantes do comportamento alimentar, os quais incluem uma complexa gama defatores nutricionais, demográficos, sociais, culturais, ambientais epsicológicos.

\section{O comportamento al imentar e seus determinantes}

O peso e a imagem corporal do indivíduo são fatores nutricionais queinfluenciam seu comportamento alimentar. Isso porque tanto o excesso de peso como a insatisfação com o próprio corpo podem motivar a realização de restrições alimentares. Calderon et al. ${ }^{2}$ verificaram que 41,3\% dos adolescentes de uma escola pública americana afirmaram comer menos do que gostariam de forma consciente para controlar o peso. Destaca-se que o início de tais práticas é observado cada vez mais precocemente. No mesmo estudo, constatou-se que $15 \%$ dos participantes relataram ter aderido a dietas restritivas antes dos 11 anos e $84 \%$ aos 14 anos.

Além da idade, outras características sociodemográficas são consideradas condicionantes do consumo alimentar, como sexo, etnia, escolaridade e estado civil. Um estudo realizado com 1.450 adultos americanos demonstrou que, tanto em homens quanto em mulheres, a escolaridade apresentava uma associação positiva com o consumo de frutas e hortaliças ${ }^{3}$. Da mesma forma, $\mathrm{H}$ avas et al. ${ }^{4}$ também constataram o papel do nível educacional como preditor do consumo desses al imentos. Nesse estudo, que envolveu 3.122 mulheres, observou-se também que o fato de ser negra, estudante, gestante ou lactante e não ser tabagista foi associado a um consumo maior de frutas e hortaliças.

Freqüentemente, a renda é considerada um delimitador das escolhas alimentares, no sentido da escassez de recursos disponíveis para permitir
0 acesso aos alimentos. Porém, são observadas outras implicações da condição financeira no comportamento alimentar. Estudo recente realizado por Drewnowski eSpecter ${ }^{5}$ mostrou que as dietas saudáveis, caracterizadas pelo maior consumo de frutas, hortaliças, grãos integrais e carnes magras, são mais caras que as dietas características do padrão ocidental, ricas em alimentos gordurosos e doces. Os autores afirmam que 0 acesso limitado a produtos de melhor qualidade nutricional podeinclusiveser analisado como um fator causal da obesidade.

Em relação aos determinantes sociais do comportamento alimentar, de Castro ${ }^{6}$ realizou uma revisão sobre o impacto de diversos fatores no tamanho das refeições consumidas. A presença de outras pessoas proporcionava um aumento significativo da quantidade consumida e do teor calórico total da refeição. 0 autor levantou duas hipóteses para explicar esse fato, denominado de "facilitação social". Primeiro, a presença de amigos ou parentes, principalmente, poderia promover uma desinibição do indivíduo, levando-o a comer mais. Em segundo lugar, a maior duração das refeições realizadas na presença de outros ocasionaria uma exposição prolongada do indivíduo aos alimentos, propiciando também um consumo maior. Verificou-se que o número de pessoas presentes em cada refeição apresentou uma correlação positiva com a quantidade consumida, variando entre um aumento de $33 \%$ na presença de uma pessoa até $96 \%$ quando sete ou mais pessoas estavam presentes ${ }^{6}$.

Do mesmo modo, fatores culturais estão envolvidos nas práticas alimentares, como o vegetarianismo e algumas religiões, que preconizam a sel eção de determinados alimentos para o consumo. 0 rigor das leis dietéticas judaicas exemplifica essa questão. Segundo a dieta kasher, é proibido o consumo de carne suína e carnes do quarto traseiro, inclusive dos animais puros, além da ingestão de ovos que contenham manchas de sangue e a mistura de laticínios com carnes?

Além disso, a política de globalização mundial facilitou o comércio de alimentos entre os países, internacionalizando práticas alimentares. U ma estratégia utilizada por grandes redes comerciais de fast foods é possi bilitar o acesso a alimentos com as mesmas características em diversos locais do mundo, de forma a isentar os indivíduos da necessidade de se real izarem novas escolhas na sua alimentação ${ }^{8}$. Entre países de continentes distintos, são observadas diferenças nas quantidades consumidas, na composição, no fracionamento da dieta e no padrão de consumo 
em relação à distribuição diurna ou noturna das refeições 6 .

Diversos estudos têm investigado a influência de aspectos psicológicos no consumo alimentar. Os itens geralmente avaliados incluem o conhecimento e as crenças sobre as características de uma alimentação saudável, a atitude frente à dieta, o reconhecimento dos seus benefícios edas barreiras encontradas para adotá-la, a disponibilidade de um suportesocial que favoreça práticas adequadas e a responsabilidade sobre compra e preparo das refeições ${ }^{9,10,11,12}$.

Uma pesquisa com amostra representativa da Inglaterra mostrou que as atitudes relacionadas a uma alimentação saudável eram elementos essenciais na diferenciação entre consumidores com alta ou baixa ingestão de frutas e hortaliças, inclusivemais importantes do queo conhecimento dos indivíduos sobre as características de uma dieta adequada ${ }^{12}$. No estudo realizado por Havas et al. ${ }^{4}$, as variáveis psicológicas associadas significativamente à maior ingestão de frutas e hortaliças foram o conhecimento correto sobre a recomendação de consumo, a menor percepção de barreiras para aumentar o consumo, as atitudes mais positivas frente à alimentação e o maior suporte social para essa prática.

Outro fator psicológico comumente avaliado em estudos de comportamento alimentar éa auto-eficácia do indivíduo. Este termo corresponde à confiança que ele tem em si mesmo em relação à sua habilidade para fazer escolhas saudáveis em determinadas situações, por exemplo, optar por frutas em vez de doces, consumir alimentos saudáveis quando está fora de casa e comer em quantidades moderadas na presença dos amigos.

Um estudo norte-americano avaliou a autoeficácia de 242 estudantes adolescentes para a realização de determinadas escolhas alimentares saudávei $s^{13}$. Foi demonstrado que maiores níveis de auto-eficácia associavam-se a um consumo menor de alimentos ricos em açúcares em ambos os sexos e menor consumo de alimentos gordurosos entre meninos. No estudo de $\mathrm{H}$ avas et al. ${ }^{4}$, também se observou uma associação entre a auto-eficácia e as práticas alimentares. Foi verificado que o escore de auto-eficácia das muIheres avaliadas relacionava-se de forma significativa ao consumo de frutas e hortaliças.

0 ambiente tem sido considerado como uma grande influência do comportamento alimentar, principalmente em estudos epidemiológicos sobre a obesidade. Na literatura, observa-se inclusive a utilização do termo "obesogênico" na des- crição do ambiente promotor da obesidade, isto é, aquele que apresenta acesso amplo efacilitado a alimentos de alta densidade energética, pobres em micronutrientes, normalmente consumidos em estabelecimentos fora do âmbito familiar ${ }^{14}$.

Considerando o exposto, é possível afirmar que o comportamento alimentar é determinado por diversas influências, que incluem aspectos nutricionais, demográficos, econômicos, sociais, culturais, ambientais e psicológicos de um indivíduo ou de uma coletividade. A interação existente entreas dimensões cognitivas e emocionais queestão envolvidas no comportamento alimentar torna-se, portanto, evidente. Verifica-se que os inquéritos alimentares utilizados atualmente referem-se apenas ao primeiro componente da referida interação, ou seja, restringem-se a uma caracterização racional da dieta, desconsiderando os demais componentes de uma prática tão complexa como a alimentar. Dessa forma, 0 estudo aprofundado do tema requer uma abordagem interdisciplinar, que inclua aspectos próprios de diferentes áreas do conhecimento científico, provenientes da N utrição, Antropologia, Economia, Sociologia e Psicologia.

\section{O conhecimento sobre comportamento alimentar em pesquisas de nutrição}

0 interesse na investigação sobre o comportamento alimentar baseia-se na possibilidade de aumentar a efetividade deintervenções nutricionais ${ }^{1}$. Acredita-se queà medida quese conhecem melhor os determinantes do comportamento alimentar, seja de um indivíduo ou de um grupo populacional, aumentem as chances de sucesso e o impacto de uma ação de promoção de práticas alimentares saudáveis $\varsigma^{15}$.

Segundo Buttriss ${ }^{11}$, 0 aspecto mais importante na promoção da saúde provavelmente é tornar o indivíduo capaz de traduzir as inúmeras informações sobre nutrição a que ele está exposto em informações práticas sobre quais alimentos deve escolher para garantir uma alimentação sau dável. Da mesma forma, o fornecimento de informações explicaria apenas racional mente uma mudança no comportamento alimentar ${ }^{1}$.

Contudo, éimportante destacar que o fornecimento de informações sobrequalquer comportamento de saúde é fundamental nas atividades educativas. 0 conhecimento contribui para sustentar ou desenvolver novas atitudes; é o componente racional necessário para motivar uma ação desejada. A pesar do fornecimento de infor- 
mações não ser um motivador incondicional das ações visadas, não há ação que ocorra sem motivação e a motivação não ocorre sem que haja a formação de uma base de experiências prévias, construídas a partir de informações recebidas ${ }^{16}$.

Stables et al. ${ }^{17}$, defato, verificaram quea consciência e o conhecimento sobre as recomendações dietéticas são preditores significativos da mudança de comportamento alimentar. Os autores conduziram um estudo nos Estados Unidos sobre a influência do conhecimento do programa 5-A-Day for a Better H ealth no consumo alimentar e observou-se que aqueles que conheciam as mensagens veiculadas pelo programa consumiam em média 1,5 porções a mais de frutas ehortaliças quando comparados aos que desconheciam tais informações.

Por outro lado, ressalta-se que o objetivo de uma intervenção nutricional não é apenas o fornecimento deinformações, mas o al cance deuma modificação no comportamento al imentar ${ }^{18}$. Este representa o grande desafio a ser enfrentado: transformar o conhecimento científico eas recomendações dietéticas em mudanças efetivas no comportamento alimentar ${ }^{19}$.

Há evidências de que intervenções nutricionais apresentam maior efetividade quando são pautadas no comportamento, nas necessidadese crenças da população-alvo. Brug et al. ${ }^{20}$ desenvolveram um programa de educação nutricional individualizado, por meio da utilização do computador, com o objetivo de melhorar o consumo alimentar de 347 trabal hadores de Amsterdã. 0 direcionamento das mensagens baseava-se nas informações coletadas num processo de triagem sobre o consumo alimentar e em três fatores psicossociais: atitudes, influência social eauto-eficácia dos participantes. Observou-se que aqueles quereceberam aintervenção individualizada apresentaram uma redução no consumo de gordura e demonstraram atitudes e intenções positivas frente à adoção de hábitos alimentares saudáveis.

Para que o indivíduo modifique de fato suas práticas alimentares, é necessária uma internalização da justificativa para uma mudança em seus costumes ${ }^{1}$. Esse processo écaracterizado pela motivação exigida para adotar uma alimentação saudável. Segundo Buttriss ${ }^{11}$, quando há uma tentativa de mudar práticas alimentares em um determinado grupo, é essencial o conhecimento sobre os fatores que motivam os indivíduos ou evitam que os mesmos realizem modificações em suas dietas.

A motivação refere-se ao processo de estimular o indivíduo a agir. Assim, são identifica- dos dois tipos de motivação para uma mudança de comportamento: a intrínseca e a extrínseca. A motivação intrínseca é aquela que surge do indivíduo, abrange seus desejos, necessidades e metas eé estabelecida a partir do desejo de se alcançar uma recompensa interna. Exemplos demotivações internas são os desejos de ter uma boa saúde, de prevenir doenças ou de perder peso. Já a motivação extrínseca é uma resposta a recompensas ou punições externas ao indivíduo einclui o suporte social recebido e possíveis recompensas materiais. As orientações médicas para o controle de uma patologia são exemplos de uma motivação extrínseca, bem como as queixas de familiares em ocasiões sociais sobre o consumo alimentar deum indivíduo, que podem atuar tanto de forma positiva como negativa, isto é, podem estimular ou prejudicar a realização de mudanças no comportamento alimentar ${ }^{3,21}$.

Estudo realizado com 1.700 consumidores do Reino Unido constatou que as principais razões que estimulavam a adoção de práticas alimentares saudáveis eram o desejo de melhorar o estado geral de saúde $(60 \%)$, motivos pessoais de saúde (20\%), perda de peso (34\%), matérias veiculadas em revistas (11\%), na televisão ou no rádio (10\%) e a pressão exercida pelo cônjuge ou parente $(9 \%)^{11}$. Observa-se que há um relato mais freqüente de fatores intrínsecos do que extrínsecos como motivação para modificações dietéticas.

Foi observado que as motivações intrínseca e extrínseca não afetam o comportamento alimentar da mesma forma. Verifica-se que apenas a motivação intrínseca é um preditor da adoção de dietas ricas em fibras, frutas e hortaliças e pobres em gordura ${ }^{3}$. Portanto, as estratégias de intervenção nutricional devem focalizar essetipo de motivação para estimular hábitos alimentares saudáveis.

\section{A percepção das práticas alimentares}

As dificuldades para se motivar os indivíduos a alterar o seu consumo alimentar têm sido muito estudadas, devendo-se considerar a gama de fatores envolvidos nesse comportamento. U ma das maiores barreiras para a prática de mudanças na dieta éa crença de que não há necessidade de alteração dos hábitos alimentares, decorrente, na maioria das vezes, de uma interpretação errada do próprio consumo. Há uma tendência dos indivíduos, especialmente entreaqueles com dietas inadequadas, serem muito otimistas quanto aos 
aspectos saudáveis de sua alimentação ${ }^{20}$. Estudo realizado com 14.331 indivíduos a partir de 15 anos d idade da União Européia mostrou que mais de $70 \%$ destes afirmaram não ser necessário alterar seu consumo alimentar, tendo em vista que julgavam sua al imentação como suficientemente saudável ${ }^{22}$. Resultados semel hantes foram encontrados na U crânia, onde 53\% da população fizeram a mesma afirmação ${ }^{23}$. Em uma amostra representativa da população adulta canadense, observou-se que $43 \%$ dos entrevistados classificaram seus hábitos al imentares como excelentes ou muito bons ${ }^{24}$.

Contudo, restam dúvidas quanto aos reais meios que a população dispõe para avaliar sua própria dieta e se as supostas alterações dietéticas realizadas para a adoção de hábitos saudáveis correspondem às recomendações dos guias alimentares. Tal fato é sustentado também pelo aumento da incidência e prevalência de doenças crônicas não-transmissí veis associadas à alimentação, quadro característico da transição nutricional que ocorre em todo o mundo ${ }^{25}$.

Esses dados representam um desafio para os profissionais de saúde em relação à busca de intervenções de sucesso, as quais sejam capazes de mobilizar os indivíduos para a adoção de práticas alimentares saudáveis. Um dos determinantes para que os indivíduos levem em consideração os comportamentos relacionados à saúde é a percepção e a convicção do indivíduo de que a ação recomendada reduziria a ameaça à sua saú$\mathrm{de}^{21,26}$. Pode-se inferir, portanto, que reconhecer a necessi dade de alteração dos hábitos al imentares é um requisito fundamental para iniciar uma mudança dietética, o queéválido tanto para adultos como para adolescentes.

\section{Model osteóricos para comportamentos de saúde}

Destaca-se a necessidade de incluir dois fatores importantes nos programas de intervenção nutricional que visam à mudança do comportamento alimentar. 0 primeiro ponto corresponde ao treinamento profissional para aquisição de habilidades técnicas que motivem os indivíduos no sentido desejado. 0 segundo aspecto éa utilização e integração de modelos teóricos no planejamento dessas ações ${ }^{21}$.

Uma teoria pode ser definida como um conjunto de conceitos, definições e proposições que apresentam uma visão si stemática de eventos ou situações de forma a explicá-los ou predizê-los.
Corresponde a uma base para o planejamento, implementação e avaliação de intervenções, possibilitando respostas ao por quê, o que e como estas devem ocorrer. Isto é, uma teoria deve, por exemplo, orientar a busca pelo por quê das recomendações de saúde pública não estarem sendo seguidas, o que os pesquisadores devem saber antes da organização dos programas intervencionais ou o que devem monitorar, medir ou comparar na avaliação de programas já existentes e como desenvolver estratégias que tenham real impacto no grupo-alvo. Teorias são, portanto, ferramentas que podem auxiliar na compreensão de diversos comportamentos de saúde e sugerir meios de alcançar mudanças nos mesmos. A pesar de diversas teorias estarem baseadas nas mesmas idéias gerais, cada teoria emprega um vocabulário único para articular fatores específicos que são considerados importantes ${ }^{27}$.

Tendo em vista a complexidade da maioria dos comportamentos de saúde, acredita-se que dificilmente uma única teoria seja suficiente para explicá-los. Portanto, são utilizados modelos teóricos, os quais correspondem a um conjunto de teorias que facilitam o entendimento de um problema específico em um contexto particular. O bserva-se na literatura científica um número crescente de teorias e modelos teóricos que envolvem comportamentos de saúde, entre os quais se destaca a utilização do modelo transteórico ${ }^{21,27}$.

\section{O modelo transteórico}

Diversos estudos ressaltam que o modelo transteórico pode ser considerado um instrumento promissor de auxílio à compreensão da mudança comportamental relacionada à saúde. 0 mesmo foi desenvolvido por dois pesquisadores norte-americanos, James O. Prochaska e Carlo DiClemente, na década de 80, mediante estudos com tabagistas. $\mathrm{Na}$ época, foi observado que muitos fumantes conseguiam abandonar o vício sem auxílio de psicoterapia, enquanto outros somente tinham sucesso com esse tipo de tratamento. 0 estímulo para as pesquisas que culminaram na elaboração do modelo foi a hipótese, posteriormenteconfirmada, de queexistiam princípios básicos que explicariam a estrutura da mudança de comportamento que ocorria na presença ou não de psicoterapia ${ }^{28}$.

Deste então, o modelo transteórico tem sido aplicado a outros comportamentos além do tabagismo, como alcoolismo, uso de drogas, manifestação de distúrbios de ansiedade e pânico, 
realização de mamografia, prática de atividade física, modo de exposição solar e para o planejamento de estratégias de prevenção de diferentes tipos de câncer, de gravidez não-planejada, de HIV/AIDS, entre outras situações ${ }^{29}$. M ais recentemente, observa-se sua utilização na área da mudança al imentar, focalizando diferentes aspectos: consumo de gordura, frutas, hortaliças, fibras e cálcio, além de estratégias dietéticas para o controle do peso e do diabetes ${ }^{30}$.

0 modelo transteórico utiliza estágios de mudança para integrar processos e princípios de mudança provenientes das principais teorias de intervenção, o que explica o prefixo "trans" desua nomenclatura ${ }^{29}$. Freqüentemente, é também denominado modelo deestágios de mudança decomportamento. De acordo com esse modelo, as alterações no comportamento relacionado à saúde ocorrem por meio de cinco estágios distintos: pré contemplação, contemplação, decisão, ação e manutenção ${ }^{28}$. Cada estágio representa a dimensão temporal da mudança do comportamento, ou seja, mostra quando a mudança ocorre e qual é seu grau de motivação para realizá-la31.

No estágio de pré- contemplação, a mudança comportamental ainda não foi considerada pelo indivíduo ou não foram realizadas alterações no comportamento e não há intenção de adotá-las num futuro próximo (considerando-se, geralmente, seis meses). Tal situação pode ser decorrente da falta de informações corretas sobre as conseqüências de seu comportamento ou refereseà situação na qual o indivíduo já realizou diversas tentativas frustradas de alterar suas atitudes e atualmente não acredita mais em sua capacidade para modificá-las de forma efetiva. Ou seja, os indivíduos nesse estágio reconhecem a solução, mas não reconhecem o problema. Estes tendem a apresentar maior resistência, pouca motivação e são classificados como não prontos para os programas de promoção de saúde ${ }^{28,29}$. Em relação ao comportamento alimentar, este estágio corresponde àqueles que não reconhecem suas práticas alimentares inadequadas ou não dispõem da motivação necessária para alterá-las.

No estágio de contemplação, o indivíduo começa a considerar a mudança comportamental. Isto é, pretende-se alterar o comportamento no futuro, mas ainda não foi estabelecido um prazo para tanto. 0 indivíduo, portanto, reconheceque o problema existe, está seriamente decidido a superá-lo, mas ainda não apresenta um comprometimento decisivo. N esse estágio, há conhecimento dos ben efícios da mudança, mas diversas barreiras são percebidas, as quais impedem a ação desejada29, 32. Refere-se, por exemplo, ao indivíduo que reconhece que tem um padrão alimentar pouco saudável, mas acredita que a falta de tempo, o preço ou o sabor desagradável de alimentos tidos como saudáveis não possibilitam a adoção de uma dieta adequada.

0 indivíduo em decisão, estágio também denominado preparação, pretende alterar seu comportamento num futuro próximo, como no próximo mês. Geralmente, após ter superado tentativas anteriores frustradas, são realizadas pequenas mudanças e um plano de ação é adotado, ainda sem assumir um compromisso sério com o mesmo ${ }^{29}$. Considerando-se uma mudança no comportamento alimentar, sugere-se que uma expressão característica desse estágio seja manifestar o seguinte desejo: "na próxima segundafeira, começarei a dieta".

Já os indivíduos em ação correspondem àqueles que alteraram de fato seu comportamento, suas experiências ou seu ambiente de modo a superar as barreiras antes percebidas. Tais mudanças são visíveis e ocorreram recentemente, como nos últimos seis meses. Trata-se de um estágio que exige grande dedicação e disposição para evitar recaídas ${ }^{29}$. Por exemplo, um indivíduo que reduziu seu consumo de alimentos gordurosos, visando à melhora do perfil lipídico, e passa a reconhecer os primeiros benefícios da modificação de suas práticas anteriores poderia ser classificado em ação.

No estágio de manutenção, o indivíduo já modificou seu comportamento e o manteve por mais de seis meses. 0 foco daqueles assim classificados é prevenir recaídas e consolidar os ganhos obtidos durante a ação. Cabe ressaltar que não se trata de um estágio estático, tendo em vista que há uma continuação da mudança de comportamento iniciada no estágio anterior ${ }^{28}$. Em relação à alimentação, poderia corresponder a um adulto que passou por uma reeducação alimentar e adotou uma dieta saudável há mais de um ano.

Há uma tendência de analisar os estágios de mudança de comportamento como uma seqüência estática e linear. Contudo, observa-se que freqüentementeindivíduos classificados em ação não conseguem manter suas estratégias na primeira tentativa, o que promoveuma nova classificação do indivíduo em estágios anteriores. Isto é, a ocorrência de recaídas é comum eleva a uma evolução dinâmica eaum delineamento em espiral do modelo de estágios de mudança ${ }^{28}$.

A classificação dos indivíduos nos estágios de mudança de comportamento é realizada por 
um algoritmo, um questionário que compreende um número limitado de perguntas reciprocamente exclusivas. A pesar das diferenças entre os al goritmos aplicados em diferentes pesqui sas, foi observado que os indivíduos classificados nos estágios mais avançados apresentavam um consumo menor de gordura e maior de frutas, hortaliças e fibras ${ }^{32,33}$.

Trudeau et al. ${ }^{3}$ demonstraram que indivíduos no estágio demanutenção consumiam 0,99 e 0,68 porções diárias a mais de frutas e hortaliças respectivamente em comparação com aqueles nos estágios de mudança alimentar anteriores ao da ação. Outro estudo realizado entre chineses moradores de Singapura investigou os fatores que influenciavam o consumo de cereais e constatouse que $89 \%$ da amostra que apresentava consumo inadequado decereaisforam classificados nos estágios anteriores aos de ação e $75 \%$ dos que apresentavam consumo adequado de grãos estavam nos estágios de ação ou de manutenção ${ }^{34}$.

Na União Européia, $52 \%$ dos indivíduos foram classificados no estágio de pré contemplação, o que, segundo de Graaf et al. ${ }^{32}$, revela quea maioria dos europeus não considerava a possibilidade de alteração dos seus hábitos alimentares para adotar práticas mais saudáveis. No mesmo estudo, observou-se que as mulheres e os indivíduos de nível educacional mais el evado tendiam a ser classificados no estágio de manutenção, enquanto que os homens e os indivíduos debaixa escolaridadeapresentavam uma tendência maior de estar nos estágios mais precoces.

\section{Limitações do uso do modelo transteórico no comportamento alimentar}

É importante destacar as dificuldades encontradas na aplicação do modelo transteórico, devido à complexidade que o tema envolve. Sabe-se que o comportamento alimentar consiste no consumo de no mínimo centenas de al imentos e bebidas. Por outro lado, o tabagismo, comportamento no qual se baseou o desenvolvimento da teoria em questão, envolve o consumo de apenas um item, o cigarro ${ }^{15,32}$. Além disso, deve-se considerar que o tratamento do tabagismo preconiza o abandono do vício, isto é, a eliminação de determinada prática. Contudo, uma intervenção nutricional não pode eliminar a prática "alimentação". D eve-se, obviamente, mantê-la, mas modificála, de modo a que esta se torne mais saudável e adequada para o indivíduo.

Outra limitação importante corresponde ao fato do modelo ter sido delineado visando à interpretação e intervenção sobre o comportamento de indivíduos; esse fato pode ser considerado um entrave para a aplicação do mesmo em atividades de educação nutricional destinadas a coletividades. Porém, estudos de intervenção baseados no modelo transteórico realizados em grupos populacionais têm mostrado resultados satisfatórios ${ }^{15,35}$.

Ressalta-se também que o modelo transteórico engloba a avaliação de outras dimensões do comportamento, além dos estágios de mudança, tais como os processos de mudança, o equilíbrio dedecisões ea auto-eficácia do indivíduo. Osprocessos de mudança possibilitam a compreensão sobre como a mudança de comportamento ocorre entre os estágios. À medida que o indivíduo modifica seu comportamento, são identificados dez processos de mudança, a saber: aumento da consciência, alívio dramático, auto-reavaliação, autolibertação, relacionamentos de auxílio, condicionamento contrário, administração de contingências, controle dos estímulos e liberação social. No equilíbrio de decisões, o indivíduo avalia os prós e os contras da mudança de comportamento. A auto-eficácia envolve a confiança que o indivíduo tem em si mesmo para superar situações de desafio em sua mudança comportamental e a habilidade de enfrentar as tentações contrárias a uma modificação saudáve|28,29,30.

Contudo, observa-se que a maioria dos estudos que utilizam o modelo transteórico no comportamento alimentar não incluem as dimensões citadas acima, restringindo-se apenas à classificação dos estágios de mudança323,33,34. I sso se deve à dificuldade de integração de todos os componentes entreos estágio ${ }^{29}$. Além disso, não foram desenvolvidos atéo momento instrumentos capazes de avaliar com precisão tais componentes do modelo quanto às diferentes práticas alimentares. Acredita-setambém na influência do fato de que a aplicação deste no comportamento alimentar é relativamente recente, o que exige 0 aprofundamento de pesquisas no tema.

Intervenções nutricionais e o uso do modelo transteórico

De Graaf et al. ${ }^{32}$ avaliaram as principais influências na escol ha al imentar entre consumidores da União Européia e observaram que os indivíduos no estágio de pré-contemplação consideravam 0 sabor dos alimentos como o fator mais importante, enquanto que no estágio de manutenção os indivíduos consideravam a saúde como fator 
principal. Isso sugere que aqueles em pré-contemplação podem ser beneficiados principalmente com mensagens edu cativas que demonstrem a importância de uma dieta saudável; já para as pessoas no estágio de manutenção, a estratégia pode ser o fornecimento de informações mais detal hadas e práticas, como receitas saudáveis e conhecimentos específicos sobre nutrição.

Asintervenções nutricionais tradicionalmente utilizadas partem do pressuposto de que os indivíduos estão prontos para a ação, isto é, para uma mudança do seu comportamento alimentar, o que tem se mostrado insustentável na maioria das situações. Acredita-se que os programas de educação nutricional possam ser beneficiados caso considerem os diferentes estágios de mudança comportamental, tendo em vista que cada um deles corresponde a diferentes atitudes e percepções perante a nutrição e a saúde. Dessa forma, o desenvolvimento de intervenções específicas para cada estágio de mudança de comportamento alimentar pode proporcionar maior eficácia quanto à motivação dos indivíduos a adotar e manter o comportamento alterado.

\section{Conclusão}

A avaliação do consumo alimentar, bem como os guias alimentares atuais, não contempla as dimensões cognitiva eemocional que estão envolvidas no comportamento alimentar. Uma abordagem holística é imprescindível para enfrentar 0 desafio de motivar os indivíduos para a adoção de uma alimentação saudável. Portanto, a ampliação do conhecimento sobre os inúmeros determinantes do comportamento alimentar é uma importante ferramenta para superar o desafio de transformar informações científicas de nutrição em mudanças reais das práticas alimentares.

Considerando-se o impacto do comportamento alimentar na saúde, verifica-se a necessidade do desenvolvimento deestratégias deintervenção nutricional de sucesso para a adoção de práticas alimentares saudáveis em nível populacional. Sugere-sequea identificação dos diferentes estágios de mudança de comportamento seja o passo inicial para tanto. Tal classificação possibilita a formulação e aplicação de programas de educação nutricional específicos, demodo a promover maior estímulo e motivação para a mudança de práticas alimentares inadequadas e propiciar uma qualidade de vida adequada em Iongo prazo. 


\section{Colaboradores}

N Toral realizou a revisão bibliográfica e redigiu a versão inicial do artigo, que se refere a parte de sua dissertação de mestrado, intitulada “Estágios de mudança de comportamento e sua relação com o consumo alimentar de adolescentes", defendida em março de2006, na Faculdade deSaúdePública da Universidade de São Paulo. B Slater contribuiu com a concepção e a revisão crítica do artigo, além de ter orientado a referida dissertação.

\section{Referências}

1. Garcia RWD. A comida, a dieta, o gosto - mudanças na cultura alimentar urbana [tese]. São Paulo (SP): Instituto de Psicologia, Universidade de São Paulo; 1999.

2. Calderon $L L, Y u C K$, Jambazian P. Dieting practices in high school students. J Am Diet Assoc 2004; 104:1369-1374.

3. Trudeau E, Kristal AR, Li S, Patterson RE. Demographic and psychosocial predictors of fruit and vegetable intakes differ: implications for dietary interventions. J Am Diet Assoc 1998; 98(12):1412-7.

4. Havas $\mathrm{S}$ et al. Factors associated with fruit and vegetable consumption among women participating in WIC. J Am Diet Assoc 1998; 98:1141-1148.

5. Drewnowski A, Specter SE. Poverty and obesity: the role of energy density and energy costs. Am J Clin Nutr 2004; 79:6-16.

6. Castro JM. Socio-cultural determinants of meal size and frequency. Br J Nutr 1997; 77 (Suppl 1):S39-S55.

7. Topel MF. As leis dietéticas judaicas: um prato cheio para a antropologia. Horizontes Antropológicos 2003; 9(19):203-222.

8. Lennernäs $M$ et al. Influences on food choice perceived to be important by nationally-representative samples of adults in the European Union. Eur J Clin Nutr 1997; 51(Suppl 2): S8-S15.

9. Cotugna N, Subar AF, Heimendinger J, Kahle L. Nutrition and cancer prevention knowledge, beliefs, attitudes, and practices: The 1987 National Health Interview Survey. J Am Diet Assoc 1992; 92:963-968.

10. Kristal AR et al. Psychosocial correlates of healthful diets: baseline results from the Working Well Study. Prev M ed 1995; 24:221-228.

11. Buttriss JL. Food and nutrition: attitudes, beliefs, and knowledge in the United Kingdom. Am J Clin Nutr 1997; 65:1985S-1995S.

12. Thompson RL, Margetts BM, Speller VM, McVey D. The Health Education Authority's health and lifestyle survey 1993: who are the low fruit and vegetable consumers? J Epidemiol Community Health 1999; 53(5):294-299.

13. Cusatis DC, Shannon BM. Influences on adolescent eating behavior. J Adolesc Health 1996; 18:27-34.

14. World Health Organization (WHO). Diet, nutrition and the prevention of chronic diseases. Geneva: Technical Report Series 916; 2003.

15. Ni M hurchu C, Margetts BM, Speller VM. Applying the stages-of-change model to dietary change. Nutr Rev 1997; 55(1 Pt 1):10-16.

16. Kilander HF. Testing health information of students and adults. J School Health 2001; 71 (8):411-413.

17. Stables GJ et al. Changes in vegetable and fruit consumption and awareness among US adults: results of the 1991 and 19975 A Day for Better Health Program surveys. J Am Diet Assoc 2002; 102(6):809-817.

18. van Weel $C$. Nutritional guidance in general practice - a conceptual framework. Eur J Clin Nutr 1999; 53(Suppl 2): S108-S111.

19. M a J, Betts NM, Horacdek T, Georgiou C, White A. Assessing stages of change for fruit and vegetable intake in young adults: a combination of traditional staging algorithms and food-frequency questionnaires. Health Educ Res 2003; 18(2):224-236. 
20. Brug J, Steenhuis I, van Assema P, de Vries H. The impact of a computer-tailored nutrition intervention. Prev M ed 1996; 25:236-242.

21. Assis M AA, Nahas MV. Aspectos motivacionais em programas de mudança de comportamento alimentar. Rev Nutr 1999; 12(1):33-41.

22. Kearney $M$ et al. Perceived need to alter eating habits among representative samples of adults from all member states of the European Union. Eur J Clin Nutr 1997; 51(Suppl. 2): S30-S35.

23. Biloukha 0 , Utermohlen V. Healthy eating in U kraine: attitudes, barriers and information sources. Public Health Nutr 2001; 4(2):207-215.

24. Reid DJ, Conrad SA, Hendricks SM. Tracking nutrition trends, 1989-1994: an update on Canadians' attitudes, knowledge and reported actions. Can J Public Health 1996; 87(2):113-118.

25. Popkin BM. The nutrition transition and obesity in the developing world. J Nutr 2001; 131(3): 871S8735 .

26. Janz NK, Becker MH. The Health Belief Model: a decade later. Health Educ Q 1984; 11(1):1-47.

27. Glanz K, Lewis FM, Rimer BK. Linking theory, research, and practice. In: Glanz K, Lewis FM, Rimer BK, editors. Health Behavior and $\mathrm{H}$ ealth Education: Theory, Research, and Practice. $2^{\text {nd }}$ ed. California: Jossey-Bass; 1996.

28. Prochaska JO, Di Clemente CC, Norcross JC. In search of how people change - applications to addictive behaviors. Am Psychol 1992; 47(9):1102-1114.

29. Prochaska JO, Redding CA, Evers KE. The Transtheoretical Model and stages of change. In: Glanz K, Lewis FM, Rimer BK. editors. Health Behavior and $\mathrm{Health}$ Education: Theory, Research, and Practice. $2^{\text {nd }}$ ed. California: Jossey-Bass; 1996.
30. Rossi SR et al. Validation of decisional balance and situational temptations measures for dietary fat reduction in a large school-based population of adolescents. Eating Behaviors 2001; 2:1-18.

31. Greene GW et al. Dietary applications of the Stages of Change M odel. J Am Diet Assoc 1999; 99 (6):673678.

32. de Graaf $C$, van der Gaag $M$, Kafatos $A$, Lennernas $M$, Kearney JM. Stages of dietary change among nationally-representative samples of adults in the European Union. Eur J Clin Nutr 1997; 51(Suppl. 2): S47-S56.

33. Glanz K, Kristal AR, Heimendinger J, Linnan L, $M$ CLerran DF. Stages of change in adopting healthy diets: fat, fiber, and correlates of nutrient intake. Health Educ Q 1994; 21(4): 499-519.

34. Ling AM, H orwath C. Defining and measuring stages of change for dietary behaviors: readiness to meet fruit, vegetable, and grain guidelines among $\mathrm{Chi}$ nese Singaporeans. J Am Diet Assoc 2000; 100(8): 898-904.

35. Frenn M, Malin S, Bansal NK. Stage-based interventions for low-fat diet with middle school students. J Pediatr Nurs 2003; 18(1):36-45.

Artigo apresentado em 08/06/2005

Aprovado em 08/12/2006

Versão final apresentada em 17/01/2007 